PREPARED FOR THE U.S. DEPARTMENT OF ENERGY, UNDER CONTRACT DE-AC02-76CH03073

PPPL-3773

PPPL-3773

UC-70

Kinetic Effects on the Stability Properties

of Field-reversed Configurations: I. Linear Stability

by

Elena V. Belova, Ronald C. Davidson, Hantao Ji, and Masaaki Yamada

January 2003

NM|

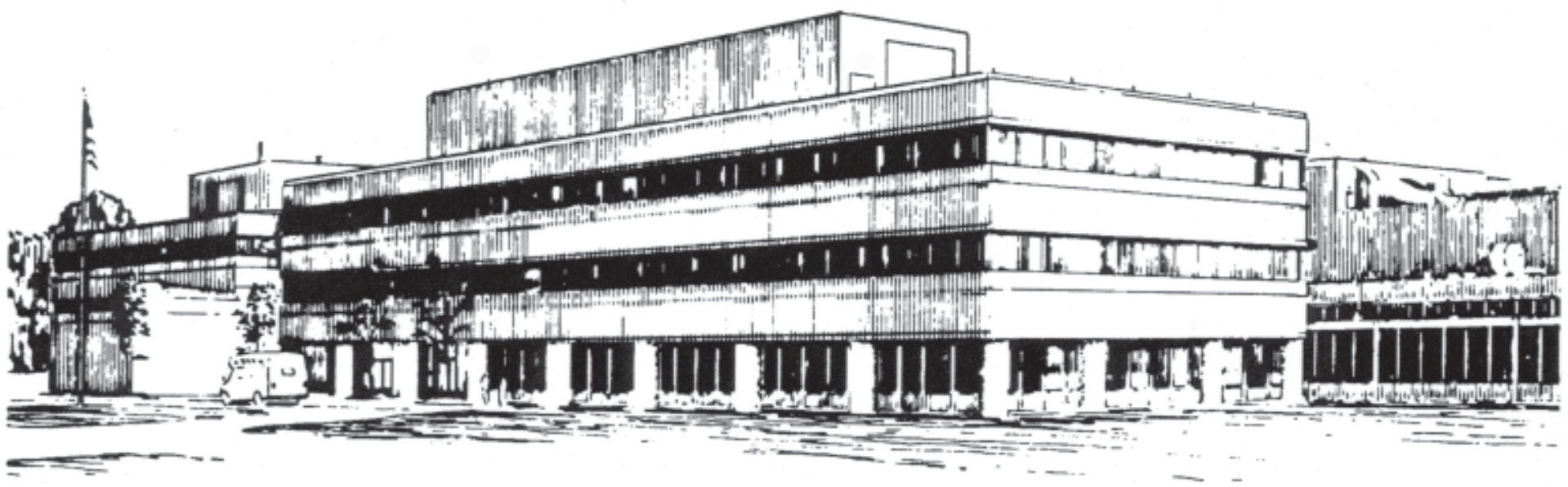

PRINCETON PLASMA PHYSICS LABORATORY PRINCETON UNIVERSITY, PRINCETON, NEW JERSEY 


\section{PPPL Reports Disclaimer}

This report was prepared as an account of work sponsored by an agency of the United States Government. Neither the United States Government nor any agency thereof, nor any of their employees, makes any warranty, express or implied, or assumes any legal liability or responsibility for the accuracy, completeness, or usefulness of any information, apparatus, product, or process disclosed, or represents that its use would not infringe privately owned rights. Reference herein to any specific commercial product, process, or service by trade name, trademark, manufacturer, or otherwise, does not necessarily constitute or imply its endorsement, recommendation, or favoring by the United States Government or any agency thereof. The views and opinions of authors expressed herein do not necessarily state or reflect those of the United States Government or any agency thereof.

\section{Availability}

This report is posted on the U.S. Department of Energy's Princeton Plasma Physics Laboratory Publications and Reports web site in Fiscal Year 2003. The home page for PPPL Reports and Publications is: http://www.pppl.gov/pub_report/

DOE and DOE Contractors can obtain copies of this report from:

U.S. Department of Energy

Office of Scientific and Technical Information

DOE Technical Information Services (DTIS)

P.O. Box 62

Oak Ridge, TN 37831

Telephone: (865) 576-8401

Fax: (865) 576-5728

Email: reports@adonis.osti.gov

This report is available to the general public from:

National Technical Information Service

U.S. Department of Commerce

5285 Port Royal Road

Springfield, VA 22161

Telephone: $1-800-553-6847$ or

(703) $605-6000$

Fax: (703) 321-8547

Internet: http://www.ntis.gov/ordering.htm 


\title{
Kinetic Effects on the Stability Properties of Field-Reversed Configurations: I. Linear Stability
}

\author{
Elena V. Belova, Ronald C. Davidson, Hantao Ji, Masaaki Yamada \\ Princeton Plasma Physics Laboratory, Princeton, NJ 08543, USA
}

\begin{abstract}
New computational results are presented which advance the understanding of the stability properties of the Field-Reversed Configuration (FRC). We present results of hybrid and two-fluid (Hall-MHD) simulations of prolate FRCs. The $n=1$ tilt instability mechanism and growth rate reduction mechanisms are investigated in detail including resonant particle effects, finite Larmor radius and Hall stabilization, and profile effects. It is shown that the Hall effect determines the mode rotation and the change in the linear mode structure in the kinetic regime; however, the reduction in the growth rate is mostly due to finite Larmor radius effects. Resonant wave-particle interactions are studied as a function of (a) elongation, (b) the kinetic parameter $S^{*}$, which is proportional to the ratio of the separatrix radius to the thermal ion Larmor radius, and (c) the separatrix shape. It is demonstrated that, contrary to the usually assumed stochasticity of the ion orbits in the FRC, a large fraction of the orbits are regular in long configurations when $S^{*}$ is small. A stochasticity condition is found, and a scaling with the $S^{*}$ parameter is presented. Resonant particle effects are shown to maintain the instability in the large gyroradius regime regardless of the separatrix shape.
\end{abstract}




\section{INTRODUCTION}

The field-reversed configuration (FRC) is a unique confinement approach that offers an attractive fusion reactor potential because of its compact and simple geometry, translation properties, and high plasma beta ${ }^{1,2}$. One of the most important issues is FRC stability with respect to low- $n$ (toroidal mode number) magnetohydrodynamic (MHD) modes. According to an empirical scaling ${ }^{3}$, based on the experimental data for prolate FRCs, stability with respect to global MHD modes is observed for $S^{*} / E \lesssim 3-4$, where $E$ is the separatrix elongation, and $S^{*}$ is a kinetic parameter which measures the number of thermal ion gyroradii in the configuration.

Despite more than two decades of effort, these results are still lacking a satisfactory theoretical explanation. Magnetohydrodynamic models $\left(S^{*} \rightarrow \infty\right.$ limit $)$ predict strong instabilities in prolate configurations $(E>1)$, particularly, with respect to the $n=1$ internal tilt mode. Simple semi-analytical estimates of finite Larmor radius (FLR) effects and Hall stabilization effects, as well as more accurate calculations employing a variational approach, give a stability threshold for $S^{*} / E \lesssim 1-2$, which is lower than the observed stability threshold by a factor of two to four, and is applicable only to elliptic configurations ${ }^{4-6}$. The discrepancy is even stronger for configurations with large $E$ and racetrack-like separatrix shapes $^{7}$. Moreover, several self-consistent numerical calculations ${ }^{8-11}$, including FLR or Hall effects, as well as a fully kinetic ion description, find that the $n=1$ tilt mode remains linearly unstable even in the highly kinetic regime: $S^{*} / E \sim 1$.

Previous hybrid simulations, utilizing a linearized version of a three-dimensional (3D)

hybrid and MHD simulation code $(\mathrm{HYM})^{10}$, have shown a significant reduction in the 
growth rates due to kinetic effects, but not complete linear stability. Recent nonlinear HYM simulations ${ }^{12}$, on the other hand, have demonstrated that the $n=1$ tilt instability can saturate nonlinearly. The unstable configuration has been shown to evolve nonlinearly into a new quasi-equilibrium with smaller $S^{*}$, larger $E$, and an increased separatrix beta, so that in the saturated state $S^{*} / E \sim 1$ and $\beta_{s} \gtrsim 0.4$. The nonlinear stabilization of the $n=1$ tilt mode explains the observation in the low $S^{*} / E$ experiments of initial $n=1$ tilt motion that does not result in a total loss of confinement ${ }^{13}$. However, the reported FRC stability for larger values of $S^{*} / E$ has not been explained so far.

Here we present the results of hybrid and two-fluid (Hall-MHD) simulation studies of the stability properties of prolate FRCs. This paper is concerned only with linear stability; the nonlinear results are presented in a companion paper. The linearized simulations are used to clarify the relative role of various competing kinetic effects, without the complications of a nonlinear model. Understanding of the linear instability mechanisms is also necessary for the understanding the nonlinear behavior of the unstable modes.

In order to assess the importance of different stabilizing factors and driving forces on the $n=1$ tilt mode, we have focussed on three kinetic effects: finite-Larmor-radius effects, the Hall effect, and resonant ions. The first two effects are stabilizing, whereas the third one can be destabilizing, and tends to obscure the FLR and Hall stabilization in fully kinetic calculations. The effects of the Hall term have been investigated in a two-fluid approximation, using a Hall-MHD version of the HYM code. Other kinetic effects have been studied using a hybrid version of the same code, in which electrons are described as a cold fluid, and a fully kinetic (particle) description is used for the ions. The hybrid version of the HYM code employs the delta-f particle simulation method, which greatly simplifies the linearization of 
the Vlasov equation. This method also allows for a detailed study of weak kinetic instabilities, which otherwise would be obscured by the large numerical noise present in conventional particle-in-cell simulations.

Two classes of equilibria have been considered: equilibria with an arbitrarily chosen pressure profile, which, for large $E$, usually correspond to a configuration with a racetracklike separatrix shape; and equilibria with large $E$ and an elliptical separatrix shape with the special pressure profile proposed by Barnes ${ }^{14}$. The methods for computing these equilibria are described in Refs. [10] and [14]. Equilibrium ion rotation is neglected.

The FRC parameters used throughout this paper are defined as follows: the separatrix elongation is the ratio of the separatrix half-length to its radius, $E=Z_{s} / R_{s}$; the kinetic parameter, $S^{*}=R_{s} / \lambda_{i}$, is the ratio of the separatrix radius to the ion skin depth, based on the maximum density, $n_{0}$; another useful kinetic parameter $\bar{s}$ is defined by

$$
\bar{s}=\int_{R_{0}}^{R_{s}} \frac{r d r}{R_{s} \rho_{i}},
$$

where $R_{0}$ is the magnetic null radius, and $\rho_{i}$ is the local ion Larmor radius. The parameter $\bar{s}$ is approximately equal to the number of ion Larmor radii between the magnetic null and the separatrix, whereas, from pressure balance $V_{t h, i} \approx V_{A}$, and therefore $S^{*} \approx R_{s} / \rho_{\text {ext }}$, which is the ratio of the separatrix radius to the ion gyroradius in the external field. For most equilibria of practical interest, the two kinetic parameters are related by $S^{*}=(4-8) \bar{s}$, depending on the elongation and shape. The characteristic Alfvén velocity $V_{A}$ is computed using the external field, $B_{\text {ext }}$, and the maximum density; and the Alfvén time is defined as $t_{A}=R_{c} / V_{A}$, where $R_{c}$ is the radius of the flux conserving shell.

The outline of this paper is as follows. Section II describes the results of the Hall- 
MHD simulations. The effects of FLR and profile effects are studied in Sections III and IV, respectively. The equilibrium characteristics and, in particular, stochasticity of ion orbits for various FRC parameters and profiles are considered in Section V, and resonant particle interactions with the $n=1$ tilt mode are discussed in Section VI. The conclusions are presented in Section VII.

\section{EFFECTS OF THE HALL TERM}

Ideal MHD models predict instability of the $n=1$ internal tilt mode in prolate FRCs with a growth rate of order $\gamma_{m h d} \approx C V_{A} / Z_{s}$, where $C \approx 1-2$ is a constant which depends on the equilibrium profile. Inclusion of the Hall term into the MHD model results in a phase shift between the perturbations of the magnetic field (frozen into the electron fluid) and the ion flow velocity, which, in turn, is expected to reduce the instability growth rate ${ }^{4,6,9}$ at low $S^{*}$.

The effects of the Hall term have been considered using a variational approach for Hill's vortex equilibrium, with a set of trial functions ${ }^{4}$. A stability condition of the form $S^{*} / E \leq 1$ has been obtained. The Hall stabilization effect has also been studied for long elliptical FRC equilibria using an expansion in the inverse elongation ${ }^{6}$. For long elliptical profiles, a similar stability condition (local) was found, and it has been argued that the global stability condition can be close to the one experimentally observed. Earlier self-consistent 3D simulations ${ }^{9}$, on the other hand, have found a reduction in the growth rate and a change in the linear mode structure, but no complete stabilization even in cases with $S^{*} / E<0.6$. The simulations in Ref. 9 were performed for FRCs with a racetrack separatrix shape, which could possibly account for the disagreement with the variational studies ${ }^{4,6}$. We were motivated to 
study the Hall-MHD stability for a class of elliptic configurations, which have been suggested to have favorable stability properties ${ }^{14}$.

To isolate the Hall effects from other kinetic effects, we have performed two-fluid (HallMHD) simulations of the $n=1$ tilt instability. The MHD version of HYM code has been modified to include the Hall term in the Ohm's law,

$$
\mathbf{E}=-\mathbf{v}_{e} \times \mathbf{B} / c+\eta \mathbf{J}
$$

where $\mathbf{v}_{e}=\left(\mathbf{J}_{i}-\mathbf{J}\right) / e n_{e}$ is the electron flow velocity, $\mathbf{J}_{i}$ is the ion current, $\mathbf{J}$ is the total current, $n_{e}$ is the plasma density, and the electron pressure has been neglected $\left(T_{e}=0\right)$. Sub-cycling in the induction equation has been used to assure numerical stability.

Calculations for an equilibrium with $E=6.25, x_{s}=R_{s} / R_{c}=0.8$, and an elliptical separatrix shape show a reduction in the instability drive for small $S^{*}$ values. Figure 1 shows the growth rate and the absolute value of the real frequency of the most unstable mode as function of the parameter $1 / S^{*}$. The unstable mode has a negative real frequency, and it rotates in the direction opposite to that of the equilibrium current (in our model, the external magnetic field has a negative sign, the equilibrium current is in the positive $\hat{\phi}$ direction, and the ion rotation has been neglected). The toroidal phase velocity of the unstable mode is about one-half of the electron equilibrium flow velocity $V_{0 e}$, and it scales approximately linearly with $1 / S^{*}$ (Fig. 1). This behavior of the real frequency is consistent with the approximation, $\omega\left(\omega-k_{\phi} V_{0 e}\right)+\gamma_{m h d}^{2}=0$, to the dispersion relation for the HallMHD system. The measured reduction of the growth rate, however, is not as strong as would follow from this simple relation, and the mode remains unstable even in cases when the real frequency is comparable to the ideal MHD growth rate. 
Figures 2 and 3 compare the linear mode structure obtained from the MHD and HallMHD simulations performed for the same equilibrium profile. It is seen that the inclusion of the Hall term modifies the structure of the most unstable mode. As $S^{*}$ decreases, the tilt mode becomes more strongly localized radially and axially. The most unstable mode in the Hall-MHD simulations with small $S^{*}$ has an extra node both in the radial and axial directions, compared to that of the MHD simulations.

Figure 1 shows that the tilt mode remains unstable even in the highly kinetic regime with $S^{*} / E=0.8$, and that the reduction in the growth rate is to a value less than one-half of the MHD growth rate. Simulations performed for elliptic equilibria with larger elongation $E=11.6$ have shown similar results - there is no significant reduction in the tilt mode growth rate for $S^{*} / E \gtrsim 1.5$. These results are in agreement with previous numerical simulations ${ }^{9}$, and demonstrate that Hall stabilization alone cannot account for the experimentally observed stability.

\section{FINITE LARMOR RADIUS EFFECTS}

Finite Larmor radius effects can be studied within a fluid description by including the gyroviscous force in the ion momentum equation. Divergence of the results at the magnetic null and X-points, where the magnetic field vanishes, can be avoided by including collisional viscosity $^{8}$. However, this description implies an FLR expansion, which is not valid in the large-Larmor-radius, kinetic regime. Thus, for $S^{*} / E=2$, and assuming that $E \sim 5$, we have $\bar{s} \approx 1.3-2$, which means that the ratio of the ion Larmor radius to the pressure gradient scale length is of the order of unity, with $\rho_{i} / L=0.5-0.8$. Moreover, even for relatively large $S^{*}$ cases, an FLR-fluid description may not be adequate for long elliptic equilibria due 
to the very sharp field line curvature near the FRC ends.

In order to separate the FLR effects from the Hall-term effects, we have chosen to perform hybrid simulations including the full ion dynamics, but turning off the Hall term in the Ohm's law. Instead of using Eq. (1), the perturbed electric field has been calculated using

$$
\delta \mathbf{E}=-\delta \mathbf{v}_{i} \times \mathbf{B}_{0} / c+\eta \delta \mathbf{J}
$$

The disadvantage of this approach is that other kinetic effects, such as parallel ion dynamics and resonant particle effects, are retained, which potentially can obscure the FLR physics at low values of $S^{*}$.

Configurations with an elliptical separatrix shape and zero ion rotation have been considered. The equilibrium ion distribution function is taken to be $F_{0}=N_{0} \exp \left(-\varepsilon / T_{0}\right)$, where $\varepsilon=m_{i} v^{2} / 2+e \varphi_{0}$ is the particle energy, $\varphi_{0}$ is the unperturbed electrostatic potential, and $T_{0}$ is the uniform ion temperature. The equilibrium current is assumed to be carried by the electrons, and from the force balance condition, en $\mathbf{E}_{0}=\nabla p_{i}=\mathbf{J}_{0} \times \mathbf{B}_{0}$.

Figure 4 shows the results of these simulations for an equilibrium with $E=4$. The normalized growth rate of the $n=1$ tilt mode (Fig. 4a) and the real frequency (Fig. 4b) are shown as compared to those obtained from full hybrid simulations that include the Hall effects. It is seen that, although the Hall term contributes to the stabilization, the reduction in the growth rate for $S^{*} \lesssim 10$ is mostly due to FLR effects. In simulations without the Hall term, the mode rotates in the positive (ion diamagnetic) direction, and the structure of the mode is similar to that of the ideal MHD model. Full hybrid simulations including the Hall term, on the other hand, show that the mode structure is similar to that of the Hall-MHD 
mode, and the real frequency of the tilt mode is negative for small $S^{*}$. For $S^{*} \sim 10$, the mode frequency is $\omega_{r} \approx-V_{A} / Z_{s}$, which is comparable to the Hall-MHD result (Fig. 1 ). The unstable mode rotates in the electron direction with a toroidal phase velocity about one-half of the electron flow velocity. For example, for $S^{*}=8$, the peak electron flow velocity and the wave phase-speed are $V_{e}=-0.42 V_{A}$ and $V_{p h}=-0.23 V_{A}$. The low- $S^{*}$ estimate for the mode rotation frequency, $\omega_{r} \approx-V_{A} / Z_{s}$, also agrees with the experimentally-measured values ${ }^{15}$.

A comparison of these results with the results in Sec. II demonstrates that the Hall effects are responsible for the the change in the linear mode structure, as well as the mode rotation in the electron direction, whereas the reduction in the tilt mode instability growth rate is caused mostly by FLR effects.

It is instructive to compare the hybrid simulation results with those based on the FLRfluid description. Self-consistent calculations of the tilt mode instability, including FLR effects through Braginskii's gyroviscosity have been carried out in Ref. 8. For $S^{*} / E=4$, the gyroviscous model predicts instability with growth rate $\gamma \approx 0.52 \gamma_{m h d}$. For the same parameters, the hybrid simulations without the Hall effects give a growth rate $\gamma \approx 0.59 \gamma_{m h d}$ [for a full hybrid simulation, $\left.\gamma \approx 0.41 \gamma_{m h d}\right]$. Therefore, both FLR models, while in the reasonable agreement with each other, fail to explain the experimentally-observed stability threshold [however, the stability is expected to be further improved by the ion sheared flows, which have not been included in the FLR models]. Another common feature of the hybrid and gyroviscous calculations, is that the tilt mode growth rate remains finite at small $S^{*}$. Since the FLR expansion is suspect at low $S^{*}$, the reasons for the tilt mode remaining unstable in the FLR-fluid description are not clear. In the fully-kinetic ion model, on the other hand, other kinetic effects such as resonant particle excitations can play a role. 


\section{PROFILE EFFECTS}

A number of MHD calculations ${ }^{10,14,16}$ have shown that the commonly assumed scaling of the tilt instability growth rate with elongation, $\gamma_{m h d} \sim 1 / E$, is applicable only to configurations with an elliptical shape. For racetrack equilibria, the growth rate becomes independent of elongation, when the elongation is large enough, with $E \gtrsim 5$, say. This difference is related to the differences in the mode structure: in elliptic configurations, the tilt mode is always a global mode with a maximum amplitude near the midplane, whereas in racetrack configurations, the tilt mode is localized near the ends, where the curvature is large. The scaling of the MHD growth rate with respect to elongation defines a condition for the transition to the kinetic regime ${ }^{7}$. In particular, FLR effects are likely to significantly modify the growth rate provided $\gamma_{m h d} / \omega^{*} \lesssim 1$, where $\omega^{*}$ is the diamagnetic frequency. This condition depends on the ratio $S^{*} / E$ when elliptic configurations are considered, but it is a function of the $S^{*}$ parameter for long racetrack equilibria. Therefore, the elliptic equilibria, while providing good agreement with observed profiles, are also more likely to fit the experimentally observed stability scaling with respect to $S^{*} / E$.

We have studied the effects of elongation in kinetic FRCs for a range of $S^{*}$ values using linearized delta-f hybrid simulations. The equilibrium ion distribution function has been taken to be the same as in Section III, and elliptical equilibria with uniformly slow $z$ variation have been studied ${ }^{14}$. Figure 5 shows the dependence of the normalized growth rate on the parameter $S^{*} / E$ for three different equilibria with elongations $E=4,6.25$, and 11.6. It is seen that for all elongations, the growth rate of the tilt mode is indeed a function of $S^{*} / E$ alone, at least when the value of $S^{*} / E$ is not too small $\left(S^{*} / E>2\right)$. (In contrast, this scaling is not observed for the more general case of arbitrary pressure 
profile and racetrack-like shape.) The vertical dashed line in Fig. 5 denotes the empirical experimentally-observed stability threshold at $S^{*} / E=3.5$. The region to the right of this line corresponds to the parameter regime where experimental stability has been reported. It is seen that linear theory, while predicting reduced instability, does not explain the observed macroscopic stability threshold.

Figure 5 demonstrates that the growth rate scaling with the $S^{*} / E$ parameter is valid everywhere except in the strongly kinetic regime with $S^{*} / E<2$. This deviation suggests that factors other than two-fluid effects are important in highly kinetic configurations.

\section{ANALYSIS OF THE PARTICLE ORBITS}

\section{A. Numerical Results}

The complexity of the ion orbits in the FRC configuration arises from the lack of a strong toroidal field, so that the magnetic field magnitude vanishes at the field null and at the X-points. It also results from a relatively large thermal ion gyroradius in the experimental configurations, where the particle gyroradius often exceeds the local radius of curvature of the magnetic field lines. This causes the breakdown of $\mu$ (magnetic moment) conservation, and the particle motion often exhibits only two invariants: energy, $\varepsilon$, and the toroidal canonical angular momentum, $p_{\phi}$. Ergodic behavior, therefore, is a common simplifying assumption in FRC and Ion Ring (IR) stability studies ${ }^{17}$. In some cases the third invariant does exist in part of the particle phase-space, and then, a fraction of the ions have regular orbits. For long FRCs, in which the radial betatron frequency is large compared to the axial betatron frequency, $\omega_{R} \gg \omega_{z}$, the third invariant related to the particle radial motion has been calculated $^{18,19}$. 
Linear stability theory for confinement systems in which particles have a full set of invariants and regular orbits is fairly well developed. For the FRC, new techniques taking into account the stochasticity of the ion orbits are needed. In order to assess the role of resonant wave-particle interactions in determining FRC stability properties, we have studied the stochasticity of ion equilibrium orbits for a range of FRC parameters and different separatrix shapes.

In the earlier studies, it has been shown that the occurrence of ergodic behavior in particle orbits in FRC configurations is very model dependent ${ }^{20}$. Here we consider prolate equilibria with elliptic and racetrack separatrix shapes and large elongations $E \gtrsim 5$. In contrast to previous work ${ }^{18,20,21}$, self-consistent equilibria are used with a non-zero equilibrium electric field (produced by the electron current through $\mathbf{E}=-\mathbf{V}_{e} \times \mathbf{B}$ ), and the statistics of the ion orbits for a distribution of ions with $F_{0}=N_{0} \exp \left(-\varepsilon / T_{0}\right)$ is considered.

For long FRCs, we have $\omega_{R} / \omega_{z} \approx 2 E \gg 1$, and the resonances between the radial and axial equilibrium motions of the particle can be neglected. The stochasticity of the ion motion in these cases is due to the end curvature of the magnetic field lines, which is especially large for long elliptic configurations. The orbits sampling the FRC end regions are likely to be stochastic, whereas those confined near the magnetic null, are likely to be regular $^{18}$. (There is a small fraction of regular orbits, trapped in the magnetic minima at the ends, but our analysis shows that these particles have little effect on the instability, therefore this class of particles is not important for our purposes.) Since the radial motion of the particles is very fast compared to all other time scales of interest, including the typical instability growth times, the radial motion "averages out", and the condition for a particle to be in resonance with the low-frequency wave is independent of $\omega_{R}$. In general form, this 
condition can be expressed $\operatorname{as}^{12}$ :

$$
\omega-n \Omega= \pm l \omega_{z},
$$

where $\omega$ is the real oscillation frequency, $n$ is the toroidal mode number, $\Omega$ is the particle toroidal rotation frequency, and $l$ is an odd or even integer, depending on the mode symmetry. This condition describes a resonance between the wave frequency in the particle frame rotating with frequency $\Omega$, and the axial betatron frequency. For the $n=1$ tilt mode, $l$ must be odd.

Figure 6a shows the typical trajectory of a periodic-orbit particle, and the time evolution of the particle toroidal and axial betatron frequencies is shown in Fig. 6b. Figure 7 contains analogous plots for a stochastic trajectory for an elliptic equilibrium with $E=6.25$ and $x_{s}=0.8$. The axial betatron frequency has been calculated by measuring the time intervals between the particle's subsequent crossing of the mid-plane $(z=0)$. The toroidal rotation frequency has been calculated as a time average of the particle's instantaneous toroidal rotation rate, and for particles with a small Larmor radius it corresponds to the toroidal drift frequency. It is seen that for a regular orbit, the characteristic frequencies, $\Omega$ and $\omega_{z}$, remain nearly constant for many periods of the particle motion (Fig. 6). For the stochastic orbit, however, there are large variations in both of these frequencies. The time dependence of $\Omega(t)$ and $\omega_{z}(t)$ allows the particle to stay in the resonance with the mode only for a short time. Therefore, a strong stochasticity of the equilibrium orbits will have a stabilizing effect on the resonantly-driven modes.

The growth rate of the resonant instability depends on the ergodic behavior of the ion orbits, but it also depends on the number of particles that can satisfy the resonance 
condition (3). Figure 8 shows the distribution of particles in the $\left(\Omega, \omega_{z}\right)$ plane, calculated for a set of elliptical equilibria with $E=6.25$ and different values of the kinetic parameter $S^{*}$. These scatter plots have been obtained by following the test particle equilibrium trajectories in a prescribed self-consistent equilibrium. The test particles have been loaded with the equilibrium distribution function $f_{0}(\varepsilon)$, and their trajectories have been followed for more than 100 Alfvén times. It is seen that for a large value of $S^{*}\left(S^{*} \approx 75\right.$ and $S^{*} / E \approx 12$, say), the distribution of particles in the $\left(\Omega, \omega_{z}\right)$ phase-space is very narrow, and very few particles can satisfy the resonant condition for the $n=1$ tilt mode. Therefore, for this large values of $S^{*}$, resonant wave-particle interactions are negligible (but the configuration is MHD unstable with $\left.\gamma \approx \gamma_{m h d}\right)$. As the value of the $S^{*}$ parameter decreases (Fig. 8b and 8c), the particle distribution widens along the $\Omega$-axis, and the number of resonant particles increases. For $S^{*}=9.4\left(S^{*} / E=1.5\right)$, a significant fraction of the ions can be in the resonance with the mode.

For present purposes, we are not concerned whether or not the particle orbit is periodic in a strict sense, but rather whether its toroidal and axial frequencies remain the same on the time scale of interest. Therefore, we define an orbit as "periodic", if the corresponding frequencies do not change significantly during the simulation time (equal to several instability growth times), and define it as a "stochastic" orbit otherwise.

The color in the scatter plots in Fig. 8 is used to mark periodic-orbit (green) and ergodicorbit (red) particles. The ergodicity of the orbits has been determined in the following way: the values of the equilibrium frequencies for each particle have been stored at four different times during the simulation, and the orbits with a significant variation in $\Omega$ or $\omega_{z}$ values have been considered to be ergodic, whereas orbits with nearly constant frequencies have 
been considered to be periodic. The criteria used in the periodicity checks are

$$
\frac{|\Delta \Omega|}{\max \{|\Omega|,\langle\Omega\rangle\}}<0.05, \quad \text { and } \quad \frac{\left|\Delta \omega_{z}\right|}{\omega_{z}}<0.01
$$

where $\langle\Omega\rangle$ is the toroidal frequency averaged over all particles. This method, although not

exact, gives a convenient description of the gross behavior of the distribution of particles.

The plots in Fig. 8 exclude particles that are not confined, or have crossed the midplane fewer than two times during the simulation.

Figure 8 shows that particles with small $\omega_{z}$ or negative $\Omega$ (rotation opposite to current) are most likely to be ergodic. It is also seen that the number of regular orbits increases as the configuration becomes more kinetic (smaller $S^{*}$ ). This is in contrast with the findings for oblate configurations, where the degree of ergodicity was found to increase with the particle energy ${ }^{20}$. Figure 9 shows the fraction of regular orbits $N_{\text {regular }}$ (out of all confined orbits) plotted as a function of the $1 / S^{*}$ parameter for elliptical equilibria with $x_{s}=0.8$, and $E=6.25$ (solid) and $E=11.6$ (dashed). It is evident that $N_{\text {regular }}$ increases almost linearly with $1 / S^{*}$, independent on the elongation. Results for racetrack equilibria with $x_{s}=0.64$ and $E=7.2$ are also shown in Fig. 9 (dotted line). For these equilibria, $N_{\text {regular }}$ is also found to increase with the $1 / S^{*}$ parameter, and for the same value of $S^{*}$ the number of particles with periodic orbits is larger than that in the elliptical configuration. In general, the fraction of the regular orbits can be as large as $60-70 \%$ of all confined particles for $S^{*} \sim 10$.

\section{B. Stochasticity Condition}

A simple condition which allows us to distinguish between regular and stochastic orbits in $\left(\varepsilon, p_{\phi}\right)$ phase-space can be derived by considering test-particle motion in the equilibrium fields. The radial profiles of the flux function $\psi$ and the electrostatic potential $\varphi$ are shown 
in Figs. 10a and 10b for an elliptical FRC with $E=6.25$ and $S^{*}=19$, where $\psi$ is defined by $\mathbf{B}=\nabla \phi \times \nabla \psi$, and $B_{\text {ext }}<0$. Particle motion in the $(R, Z)$-plane with constant $p_{\phi}$ and $\varepsilon$ is equivalent to the motion in the effective potential $V_{\text {eff }}(R, Z)$. For normalized values $\left(\varepsilon \rightarrow m_{i} V_{A}^{2}\right.$, and $\left.p_{\phi} \rightarrow m_{i} V_{A}^{2} / \omega_{c i}\right)$, we have $p_{\phi}=R v_{\phi}-\psi$, and $\varepsilon=\left(v_{R}^{2}+v_{Z}^{2}\right) / 2+V_{e f f}$, where

$$
V_{e f f}(R, Z)=\frac{\left(p_{\phi}+\psi\right)^{2}}{2 R^{2}}+\varphi
$$

Depending on the value of $p_{\phi}$, three different types of $V_{\text {eff }}$ are possible. For $p_{\phi}<0$ (Fig. 10c), the effective potential does not have a minimum inside the separatrix, and the particle cannot be confined. For $0<p_{\phi}<\left|\psi_{0}\right|$ (Fig. 10d), the potential has a double-well structure with a central maximum with value $V_{0} \approx V\left(R_{0}, 0\right)=\left(p_{\phi}+\psi_{0}\right)^{2} / 2 R_{0}^{2}$, where $\psi_{0}$ is the flux value at the magnetic null $\left(\psi_{0}<0\right)$. For $p_{\phi} \geq\left|\psi_{0}\right|$ (Fig. 10e), the potential has a single-well structure without a central maximum. In the double-well case, the particle is confined away from the center (magnetic null), if its energy is smaller than $V_{0}$. The orbit in this case is confined between two ellipses in the $(R, Z)$ plane, and is similar to that shown in Fig. 7a. In other cases, $p_{\phi} \geq\left|\psi_{0}\right|$ or $p_{\phi}<\left|\psi_{0}\right|$ with $\varepsilon>V_{0}$, the particle orbit can pass near the center, similar to the orbit shown in Fig. 6a.

We conclude that orbits confined in a double-well, away from the center, will be ergodic, whereas the orbits passing through the center will be regular, for the following reason. In the one-dimensional (1D) limit $(E \rightarrow \infty)$, an orbit with $\varepsilon<V_{0}$ is confined in one of the wells to the right or to the left of the center (Fig. 10d), and it is regular. The small 2D corrections $(\sim 1 / E)$ will qualitatively change the orbit behavior, allowing the particle to jump from one well to another at the ends of the configuration, and therefore, introducing the stochasticity. In contrast, for the particles passing trough the center, the 1D well will 
become an elongated 2D well when $E$ is finite, but their orbits will remain regular, because for these orbits adiabatic invariance theory based on the $\omega_{z} / \omega_{R} \sim 1 / E$ expansion is applicable.

Therefore, a condition for a particle orbit to be regular can be written as $\varepsilon>V_{0}$, or

$$
\left|\psi_{0}\right|+R_{0} \sqrt{2 \varepsilon}>p_{\phi}>\left|\psi_{0}\right|-R_{0} \sqrt{2 \varepsilon}
$$

where the upper bound for $p_{\phi}$ is simply the condition for an orbit to exist (i.e. $\varepsilon>\min \left(V_{\text {eff }}\right)$ ), and the lower bound corresponds to a transition boundary between the regular and ergodic orbits. (The above condition is not applicable for end-trapped particles, or for very low energy particles, for which $\mu$ is conserved, which are not the cases considered here).

Figure 11 shows the particle distribution in the $\left(\varepsilon, p_{\phi}\right)$ plane, where the regular-orbit particles are marked in green, and the ergodic ones are in red. It is seen that the condition in Eq. (6) describes the boundary between the regular and ergodic orbits remarkably well. Some inaccuracy is associated with the estimate $V_{0} \approx V_{e f f}\left(R_{0}, 0\right)$, and is also due to a finite simulation time, which does not allow a distinction between very slow regular particles and the stochastic particles when $\varepsilon \approx V_{0}$. Note that the boundaries of the particle distribution in $\left(\varepsilon, p_{\phi}\right)$ space in Fig. 11 are due to $\varepsilon>\min \left(V_{e f f}\right)$ and the confinement condition. Similar results and a good agreement with the condition in Eq. (6) have also been obtained for racetrack equilibria with $E=7.2$ and $S^{*} \gtrsim 10$.

Now it is evident why the fraction of regular orbits is larger for smaller values of $S^{*}$ (see Fig. 9). Since the value of $\left|\psi_{0}\right|$ scales as $\sim R_{0}^{2}$, and for the class of elliptical equilibria $\left|\psi_{0}\right| \approx$ $B_{\text {ext }} R_{0}^{2} / 4$, the condition in Eq. (6) can be written as $\left|p_{\phi} /\right| \psi_{0}|-1|<4 \sqrt{2 \varepsilon} / R_{0} \approx 8 \sqrt{\varepsilon} / S^{*}$ (in dimensionless variables). Therefore, the width of the regular-orbit region in the $p_{\phi}$-direction in Fig. 11, and approximately the fraction of regular orbits, are proportional to $1 / S^{*}$. 
The regularity condition in Eq. (6) is independent of the value of the electrostatic potential, because $\varphi$ vanishes at the magnetic null $\varphi\left(R_{0}\right)=0$. The equilibrium electric field has been found to have little effect on the confinement and ergodicity of the ion orbits for $S^{*}>10$. Its effect becomes significant when the value of $\varphi$ at the separatrix is comparable

to the first term in Eq. (5), i.e., for $S^{*}<8 \sqrt{4 \pi p_{0} / B_{0}^{2} \ln \left(p_{0} / p_{s}\right)} \approx 8$. For smaller $S^{*}$, the structure of the effective potential is modified by the equilibrium electric field, in a such a way that (a) for $p_{\phi}<0, V_{\text {eff }}$ has a minimum between the magnetic null and the separatrix at $R_{0}<R<R_{s}$; (b) for $0<p_{\phi}<\left|\psi_{0}\right|$, the relative value of the central maximum $V_{0}$ is reduced; and (c) the double well becomes non-symmetric with smaller value of the $\min \left(V_{\text {eff }}\right)$ on the outer side, $R>R_{0}$. This results in a new type of the particle orbit, which is confined away from the magnetic null, on the outer side of the configuration (i.e., at $R>R_{0}$ ), and which is found to be regular. The number of the ergodic orbits reduces compared to that predicted by the regularity condition in Eq. (6). Therefore, we find that for $S^{*}<8-10$, the equilibrium electric field improves particle confinement, and further reduces the stochasticity of the ion orbits.

\section{RESONANT PARTICLES EFFECTS}

Numerical simulations of the tilt instability for various FRC equilibria and separatrix shapes have shown that common to all cases is the change of the instability from a reactive one (with $\gamma \gg|\omega|$ ), to a weakly unstable one with $\gamma /|\omega| \ll 1$, as the value of $S^{*}$ decreases. The linear growth rate is reduced by a factor 5 to 10 compared to the MHD growth rate for $S^{*} / E \sim 1$, but it nonetheless remains finite ${ }^{10}$.

It is well known that the Vlasov-fluid (Maxwellian) plasma has the same marginal sta- 
bility condition as the MHD model, and therefore, that FLR effects cannot totally stabilize, but can only reduce the growth rates of the unstable modes ${ }^{22,23}$. The residual instability is due to resonant-particle destabilization of FLR-stabilized MHD modes. For the FRC equilibria considered here, the $n=1$ MHD mode with $\omega<0$ (in the ion frame) is a negativeenergy wave, which can be driven unstable by Landau damping on the resonant ions when $\partial f_{0} / \partial \varepsilon<0$. The growth rate of this resonant instability depends on the number of resonant particles, the slope of the ion distribution function $f_{0}$, and the stochasticity of the ion orbits.

Analysis of the particle orbits (Section V) has demonstrated that, contrary to the usually assumed stochasticity of the ion orbits in the FRC, a large fraction of the orbits (up to 60\%) can be regular in elongated kinetic configurations with $E>5$ and $S^{*} \lesssim 20$. Since a significant number of particles also can satisfy the resonant condition in Eq. (3), resonant wave-particle interactions are likely to play an important role in determining $n=1$ tilt mode stability properties at low $S^{*}$.

The linearized hybrid simulations presented here employ the delta-f method for numerical noise reduction. In this method, the equilibrium ion distribution function $f_{0}$ is assumed to be known analytically, and the equation for the perturbed distribution function $\delta f=f-f_{0}$ is integrated along the equilibrium particle trajectories. Each simulation particle is assigned a weight $w=\delta f / f$, which is evolved in time using the equation:

$$
\frac{d w}{d t}=-e(\mathbf{v} \cdot \delta \mathbf{E}) \frac{\partial\left(\ln f_{0}\right)}{\partial \varepsilon}
$$

Large-weight particles indicate regions in the phase space, where the change in the ion distribution function is the largest, and where the resonances are likely to occur.

Simulation results show that particles with the largest values of $w$ usually have regular 
orbits, and their toroidal and axial betatron frequencies satisfy the condition in Eq. (3). Figure 12 shows scatter plots of particles in the $\left[w=\delta f / f,(\Omega-\omega) / \omega_{z}\right]$ plane obtained in three simulations with an elliptical separatrix with $E=6.25$, and different values of $S^{*}$. It is evident that the resonances occur when the frequency ratio is an odd integer with $(\Omega-\omega) / \omega_{z}=1,3,5$. The resonances are not seen at negative values of $(\Omega-\omega)$, because most of these orbits are stochastic (Fig. 8). Also, in agreement with the results in Section V, for large $S^{*}$ (MHD-like regime, Fig. 12a), very few particles are in resonance with the mode. As the configuration size $\left(S^{*}\right)$ reduces, the particle distribution in frequencies broadens (Fig. 8), and the number of particles in resonance increases (Fig. 12b,c).

Table 1 shows the values of the characteristic time scales normalized to the Alfvén time $t_{A}=R_{c} / V_{A}$ for the set of simulations shown in Fig. 12. It is evident that, in general, the time scales associated with particle equilibrium motion are longer than the typical instability growth times. The three time scales become comparable when $S^{*}$ is small.

The resonance condition in Eq. (3) can be understood as follows. The perturbed distribution function [or $w$ in Eq. (7)] is proportional to the change in the particle energy, which is proportional to $(\mathbf{v} \cdot \delta \mathbf{E}) \approx v_{z} \delta E_{z}$. For the $n=1$ tilt mode, $\delta E_{z}$ is symmetric relative to the midplane $z=0$, and it can be written as $\delta E_{z}=\delta \hat{E}_{z}\left(z^{2}\right) \exp (-i \omega t+i n \phi)$, where the radial dependence is assumed to be averaged out. Therefore, the time dependence of $\delta E_{z}$ along a periodic orbit with $z \approx z_{0} \sin \left(\omega_{z} t\right)$ and $\phi \approx \Omega t$ can be expanded as

$$
\delta E_{z}=\sum_{k} \delta E_{z, k} \exp \left[-i\left(\omega-n \Omega-2 k \omega_{z}\right) t\right]
$$

where $k$ is an integer. For a particle to be in resonance with the mode, the phase of the product of $v_{z}=d z / d t$ and $\delta E_{z}$ must be constant. This results in Eq. (3) with $l=2 k+1$, 
corresponding to an odd value of $l$. [Note that for a mode with opposite symmetry, i.e., with antisymmetric $\delta E_{z}$, the condition Eq. (3) is the same, but the resonances occur for even values of $l$. Such modes are seen in the racetrack configurations, and have been called "even" modes, based on the $V_{R}$ symmetry $^{10}$.] The condition in Eq. (3) generalizes the betatron resonance condition given by Finn and $\operatorname{Sudan}^{24}$ for ion rings.

The importance of the resonances is also seen from energy balance, which shows that a relatively small number of resonant particles (a few percent) contributes significantly to the total energy balance. First, it can be shown that for $t \gg 1 / \gamma$, the linear growth rate is calculated to be

$$
\gamma=\frac{-e \sum_{m} w_{m}(\mathbf{v} \cdot \delta \mathbf{E})_{m} \partial \ln f_{0} / \partial \varepsilon}{\sum_{m} w_{m}^{2}}
$$

where $\sum_{m}$ denotes summation over all simulation particles. The numerator in Eq. (9) is proportional to $\int \delta \mathbf{j}_{i} \cdot \delta \mathbf{E} d^{3} \mathbf{x}$, and the denominator is proportional to $\Delta E=T_{0} / 2 \sum_{m} w_{m}^{2}$, which plays the role of the perturbed particle energy, and can also be written as $\Delta E=$ $\left(T_{0} / 2\right) \int \delta f^{2} / f_{0} d^{3} \mathbf{x} d^{3} \mathbf{v}$, where $f_{0} \sim \exp \left(-\varepsilon / T_{0}\right)$ with $T_{0}=$ const has been used. Assuming that the resonant particles are those with large weights, for example with $|w| \geq 0.3 \max (w)$, their number and contribution into $\gamma$ and $\Delta E$ can be estimated. In the simulations with $E=$ 6.25 and $S^{*}=8-10$, the number of large-weight particles is $\gtrsim 4-4.5 \%$, and their contribution to the energy balance is larger than 46-50\%. The expression in Eq. (9) can also be used to determine the effects of various groups of particles on the instability. We have found, in particular, that the contributions of the particles on open field lines (non-confined orbits), as well as of the end-trapped particles, were negligible.

The effects of elongation and separatrix shape on the tilt mode have been discussed in 
Section IV, where it was found that the growth rate depends only on the $S^{*} / E$ ratio when the equilibrium is elliptical and provided that $S^{*} / E \gtrsim 2$. In the more strongly kinetic cases, the growth rate depends on both $E$ and $S^{*}$, and for a given $S^{*} / E$ value, $\gamma$ is smaller for the longer configuration (Fig. 5). This result can be understood by assuming that for $S^{*} / E<2$ the instability is a resonantly-driven one. Then a larger $E$ implies a larger $S^{*}$ value, and for a larger $S^{*}$ both the number of regular orbits and the number of resonant particles are reduced (Section V); therefore, the configuration is less unstable. Thus for $S^{*} / E=1.2$, for example, the number of regular orbits in the configuration with $E=11.6$ is nearly twice as small as for $E=6.25$ (Fig. 9), and accordingly, the value of the growth rate is also almost twice as small (Fig. 5). It appears that long elliptical equilibria have better stability properties, not only in the fluid regime ${ }^{6}$, but even more so in the kinetic regimes $\left(S^{*} / E<2\right)$.

We have studied the effects of the racetrack separatrix shape on the resonant interactions and stability of configurations with $E=7.2$. Figure 13 shows a scatter plot of particles in the $\left[w,(\Omega-\omega) / \omega_{z}\right]$ plane from the simulations performed for $S^{*}=8$. A comparison with Fig. 12 (elliptical equilibria) shows that the particle distribution in equilibrium frequencies is much wider for the racetrack configuration, and therefore a large number of resonances are possible. This appears to be a typical feature of racetrack equilibria, which is related to relatively larger elongations of the internal flux surfaces in racetrack FRCs. For the simulation shown in Fig. 13, the calculated growth rate of the tilt mode is $\gamma=0.13 \gamma_{m h d}$, and we estimate the fraction of resonant particles to be about $4 \%$, with a corresponding contribution to the total particle energy change of about $63 \%$. For this equilibrium, in addition to the $n=1$ tilt mode, another mode with $n=1$, but different symmetry ("even mode" ${ }^{10}$ ) has been found to be unstable with a comparable growth rate. Since multiple resonances [i.e., with many 
l-values in Eq. (3)] are possible in the racetrack configuration, we conclude that in the kinetic regime such configurations are more unstable than the elliptical ones.

A resonant instability can be avoided, if the equilibrium ion distribution function is flattened in the region of the phase space where the resonances occur. This resonant region can be determined by considering the distribution of resonant particles in the $\left(\varepsilon, p_{\phi}\right)$ plane. Figure 14 shows a scatter plot of the simulation particles with large weights, $|w|>0.4 \max (w)$, obtained in a hybrid simulation with $E=6.25$ and $S^{*}=10$ and an elliptical separatrix. Here the value of $l$ corresponds to that in the resonance condition in Eq. (3). It is evident that all of the resonant orbits are regular [i.e., satisfy the condition in Eq. (6)]. The resonances are also highly localized in phase-space, especially the primary one with $l=1$, which shows an approximately linear relation between the resonant $\varepsilon$ and $p_{\phi}$ values. We have found that such is not the case in the racetrack configuration, where the distribution of resonant particles in $\left(\varepsilon, p_{\phi}\right)$ is much broader, which probably implies that the corresponding equilibrium frequencies (and therefore the resonance condition itself) depend on the value of the third invariant of motion.

So far we have studied FRC kinetic stability properties assuming a Maxwellian ion distribution function $f_{0}$. For the case shown in Fig. 14, a small change in $f_{0}$ will be sufficient to stabilize the mode. Therefore, a linearly-stable, small- $S^{*} / E$ configuration is possible for a non-Maxwellian $f_{0}$. The localization of the resonant particles in phase space also suggests that the instability can saturate nonlinearly through a modification of the initially unstable distribution function. This saturation has indeed been observed in hybrid simulations with $E=4$ and an elliptical separatrix, as reported previously ${ }^{12}$. 


\section{SUMMARY AND CONCLUSIONS}

We have studied the importance of different stabilizing effects and driving forces on the linear stability behavior of the $n=1$ tilt mode. Two-fluid simulations have demonstrated that Hall stabilization alone cannot account for the experimentally-observed stability regardless of the equilibrium profiles. It has been shown, at low $S^{*}$, that the Hall effects are responsible for the change in the linear mode structure, as well as the mode rotation, whereas the reduction in the growth rate is caused mostly by FLR effects. In the kinetic regime, the unstable mode is shown to rotate in the electron direction (assuming zero ion rotation) with a toroidal phase velocity equal to about one-half of the electron velocity. The real oscillation frequency is measurable in experiments, and can serve as an additional diagnostic of the kinetic tilt instability.

Hybrid simulations, performed for elliptical equilibria with uniformly slow $z$ variation ${ }^{14}$ and a wide range of $E$ values $(E=4-12)$, have shown for this class of equilibria that the tilt mode growth rate is a function of $S^{*} / E$ parameter alone. This supports the empirical stability scaling ${ }^{3}$ with the parameter $S^{*} / E$, which is based on experimental data, and it also suggests that this class of equilibrium profiles is realized in the experiments. The scaling $\gamma=\gamma\left(S^{*} / E\right)$ is valid for the two-fluid regime with $S^{*} / E>2$. In more highly kinetic regimes (i.e., $S^{*} / E<2$ ), the effects of resonant ions become important, and the kinetic growth rate $\gamma / \gamma_{m h d}$ is shown to reduce with elongation.

Analysis of the particle orbits in long configurations for different values of $S^{*}$ have produced a new and unexpected result. Contrary to the large degree of stochasticity of the ion orbits usually assumed in analyses of the FRC, we have found that a significant fraction of orbits (up to $60-70 \%$ of all confined orbits) is regular in configurations with 
$E \gtrsim 5$ and $S^{*} \lesssim 20$. Although the magnetic moment is not conserved for most of these orbits, another adiabatic invariant, based on the smallness of the $\omega_{z} / \omega_{R}$ ratio, is conserved. A regularity condition has been found, and it has been shown to agree with the numerical results remarkably well. Furthermore, the number of regular orbits has been demonstrated to scale approximately linearly with $1 / S^{*}$ (Fig. 9), so that the number of regular orbits increases from $\lesssim 10 \%$ to $\sim 60 \%$ as the configuration size is reduced from $S^{*} \approx 80$ to $S^{*} \approx 10$.

The results in this paper also show that ergodic behavior is not a suitable assumption for long kinetic FRCs with $S^{*} \lesssim 20-30$, and in the most general case the ion distribution function depends on all three integrals of motion. This conclusion is different from previous findings for field-reversed ion ring systems ${ }^{20}$, where the orbits become ergodic as the particle energy is increased. However, in these systems $E \sim 1$, and the ergodic behavior results from an overlap of the islands, corresponding to low-order resonances between the radial and axial motion. In the present case, such resonances can be neglected, and the ergodic behavior is due to the large curvature of the magnetic field lines near the FRC ends.

A large number of regular orbits in kinetic FRCs suggests that resonant wave-particle interactions play a significant role in determining stability properties. A resonance condition has been obtained that is suitable for MHD modes of arbitrary symmetry, which generalizes the betatron resonance condition of Finn and Sudan ${ }^{24}$. For a Maxwellian equilibrium distribution and low values of $S^{*}$, it has been shown that a large number of ions can be in resonance with the $n=1$ tilt mode, regardless of the FRC equilibrium profiles. The analysis of energy balance has demonstrated that the resonant particles are responsible for the instability drive in cases where the FLR and Hall effects stabilize the MHD-unstable modes. The localization of the resonant particles in phase space suggests that the instability will 
saturate nonlinearly through modification of the initially unstable distribution function. A linearly stable, small $S^{*} / E$ configuration is, therefore, possible for a non-Maxwellian $f_{0}$.

In this paper, we have investigated the linear stability properties of prolate FRCs. The results of nonlinear simulations, particularly, the nonlinear evolution of the tilting instability in large- $S^{*}$ FRC configurations, and the nonlinear saturation of the instability for low values of $S^{*}$, will be discussed in a companion paper. 


\section{ACKNOWLEDGMENTS}

The authors acknowledge the benefit of many useful discussions with D. C. Barnes, and thank him for providing the equilibrium data for long elliptical separatrix FRCs. This research was supported by the U.S. Department of Energy under Contract No. DE-AC02$76 \mathrm{CH} 03073$. 
${ }^{1}$ M. Tuszewski, Nucl. Fusion 28, 2033 (1988).

${ }^{2}$ A. L. Hoffman, L. N. Carey, E. A. Crawford et al., Fusion Technology 23, 185 (1993).

${ }^{3}$ M. Tuszewski, D. C. Barnes, R. E. Chrien, J. W. Cobb, D. J. Rej, R. E. Siemon, D. P. Taggart, B. L. Wright, Phys. Rev. Lett. 66, 711 (1991).

${ }^{4}$ A. Ishida, H. Momota and L. C. Steinhauer, Phys. Fluids 31, 3024 (1988).

${ }^{5}$ L. C. Steinhauer and A. Ishida,, Phys. Fluids B 2, 2422 (1990).

${ }^{6}$ D. C. Barnes, Phys. Plasmas 9, 560 (2002).

${ }^{7}$ N. Iwasawa, A. Ishida and L. C. Steinhauer, Phys. Plasmas 7, 931 (2000).

${ }^{8}$ N. Iwasawa, A. Ishida and L. C. Steinhauer, Phys. Plasmas 8, 1240 (2001).

${ }^{9}$ R. D. Milroy, D. C. Barnes, R. C. Bishop, R. B. Webster, Phys. Fluids B 1, 1225 (1989).

${ }^{10}$ E.V. Belova, S. C. Jardin, H. Ji, M. Yamada, R. M. Kulsrud, Phys. Plasmas 7, 4996 (2000).

${ }^{11}$ Yu. A. Omelchenko, Phys. Plasmas 7, 1443 (2000).

${ }^{12}$ E. V. Belova, S. C. Jardin, H. Ji, R. M. Kulsrud, W. Park, M. Yamada, "Global Stability of the Field Reversed Configuration", to appear in Plasma Physics and Controlled Nuclear Fusion Research, Proceedings of the 18th International Conference, Sorrento, 2000, (International Atomic Energy Agency, Vienna, 2000).

${ }^{13}$ M. Tuszewski et al.,Phys. Fluids B 3, 2856 (1991). 
${ }^{14}$ D. C. Barnes, Phys. Plasmas 8, 4856 (2001).

15 T. P. Intrator (private communication).

${ }^{16}$ N. Iwasawa, A. Ishida and L. C. Steinhauer, J. Phys. Soc. Japan, 69, 451 (2000).

17 J.M. Finn and R. N. Sudan, Phys. Rev. Lett. 10, 695 (1978).

18 J-S. Kim and J. R. Cary, Phys. Fluids 26, 2167 (1983).

${ }^{19}$ H. E. Mynick, Phys. Fluids 23, 1888 (1980); 23, 1897 (1980).

${ }^{20}$ J.M. Finn, Plasma Phys. 21, 405 (1979).

${ }^{21}$ A. H. Glasser and S. A. Cohen, Phys. Plasmas 9, 2093 (2002).

${ }^{22}$ C. E. Seyler and J. P. Freidberg, Phys. Fluids 23, 331 (1980).

${ }^{23}$ H. Tasso, in Plasma Physics and Controlled Nuclear Fusion Research, (International Atomic Energy Agency, Vienna, 1977), Vol. III, p. 371.

${ }^{24}$ J.M. Finn and R. N. Sudan, Phys. Fluids 22, 1148 (1979). 
TABLE I. Characteristic time scales obtained from the hybrid simulations shown in Fig. 12

\begin{tabular}{lccr}
\hline \hline$S^{*}$ & $T_{\beta}=2 \pi /\left\langle\omega_{z}\right\rangle\left[t_{A}\right]$ & $T_{\Omega}=2 \pi /\langle|\Omega|\rangle\left[t_{A}\right]$ & $1 / \gamma\left[t_{A}\right]$ \\
\hline 80 & 10.5 & 30. & 2.4 \\
40 & 10.1 & 22.8 \\
20 & 10.5 & 11.4 & 6.8 \\
10 & 11. & 7.8 & 10.9 \\
\hline \hline
\end{tabular}




\section{FIGURE CAPTIONS}

FIG.1. Growth rate and negative real frequency of the tilt mode from Hall-MHD simulations with $E=6.25$ and $x_{s}=0.8$; both quantities are normalized to $\gamma_{0}=V_{A} / Z_{s}$.

FIG.2. Linear structure of the most unstable mode from MHD and Hall-MHD simulations of the elliptical equilibrium with $E=6.25$. Vector plots of poloidal velocity; in Hall-MHD simulations $S^{*}=5$.

FIG.3. Contour plots of axial velocity at the midplane for the same simulations as in Figure 2.

FIG.4. Hybrid simulations with and without the Hall term, for elliptical equilibria with $E=4$. Both the growth rate (a) and the real frequency (b) are normalized to $\gamma_{0}=V_{A} / Z_{s}$.

FIG.5. Normalized growth rates obtained from linear hybrid simulations of the $n=1$ tilt mode instability for three different elliptic FRC equilibria with $\mathrm{E}=4,6.25$, and 11.6.

FIG.6. Plots of (a) the poloidal projection of the trajectory of the periodic-orbit particle; and (b) the time evolution of the particle toroidal and axial frequencies.

FIG.7. Plots of (a) the poloidal projection of the trajectory of the stochastic-orbit particle; and (b) the time evolution of the particle toroidal and axial frequencies.

FIG.8. Scatter plots of the particle distribution in the toroidal rotation and axial betatron frequencies $\left(\Omega, \omega_{z}\right)$ for three elliptical equilibria with $E=6.25$ and different values of $S^{*}$. The solid lines correspond to the resonances $(\Omega-\omega) / \omega_{z}=1$ and $(\Omega-\omega) / \omega_{z}=3$. Color is used to mark the regular-orbit (green) and ergodic-orbit (red) particles. All frequencies are normalized to the ion cyclotron frequency in the external field.

FIG.9. Plot of the fraction of regular orbits versus $1 / S^{*}$ for elliptical equilibria with $E=6.25$ (solid line) and $E=11.6$ (dashed line), and for racetrack equilibria with $E=7.2$ 
(dotted line).

FIG.10. Radial profiles at the midplane for the normalized (a) flux, (b) electrostatic potential, and (c)-(e) effective potential $V_{\text {eff }}$ for different values of the toroidal angular momentum corresponding to (c) $p_{\phi}=0.1 \psi_{0},(\mathrm{~d}) p_{\phi}=\left|\psi_{0}\right| / 3$ (solid) and $p_{\phi}=\left|\psi_{0}\right| / 2$ (dashed), and (e) $p_{\phi}=\left|\psi_{0}\right|$ (solid) and $p_{\phi}=1.5\left|\psi_{0}\right|$ (dashed). Here $\psi_{0}$ is the value of flux at the magnetic null, $R_{0}=0.7 R_{s}$.

FIG.11. Scatter plot of the particle distribution in $\left(\varepsilon, p_{\phi}\right)$ phase-space obtained from the simulation shown in Fig. 8b. Color is used to mark the regular-orbit (green) and ergodicorbit (red) particles. The solid line corresponds to the condition in Eq. (6). Dimensionless variables are used.

FIG.12. Scatter plot of the particle distribution in weight $w=\delta f / f$ (arbitrary units) and frequency $(\Omega-\omega) / \omega_{z}$, obtained in the linearized hybrid simulations for the same parameters as in Fig. 8.

FIG.13. Scatter plot of the particle distribution in weight $w=\delta f / f$ (arbitrary units) and frequency $(\Omega-\omega) / \omega_{z}$, obtained in the linearized hybrid simulations for the racetrack configuration with $E=7.2$ and $S^{*}=8$.

FIG.14. Scatter plot of resonant (large-weight) particles in $\left(\varepsilon, p_{\phi}\right)$ phase-space obtained in hybrid simulations with $E=6.25$ and $S^{*}=10$. The dashed line marks the boundary between regular and stochastic orbits as defined in Eq. (6). 


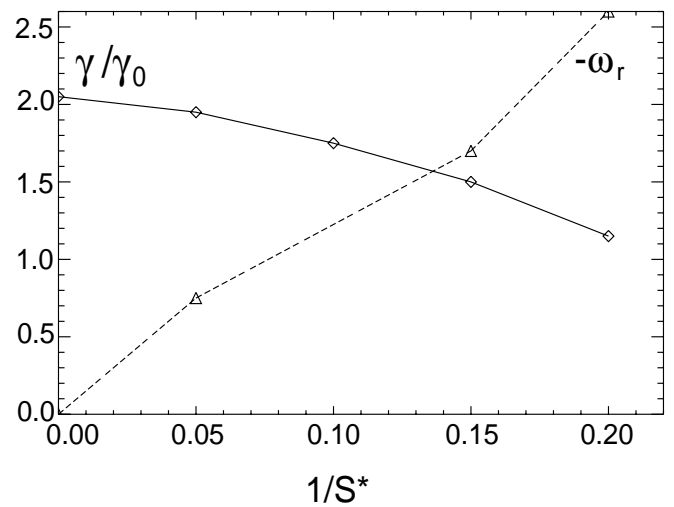

Figure 1.

Belova, Physics of Plasmas 


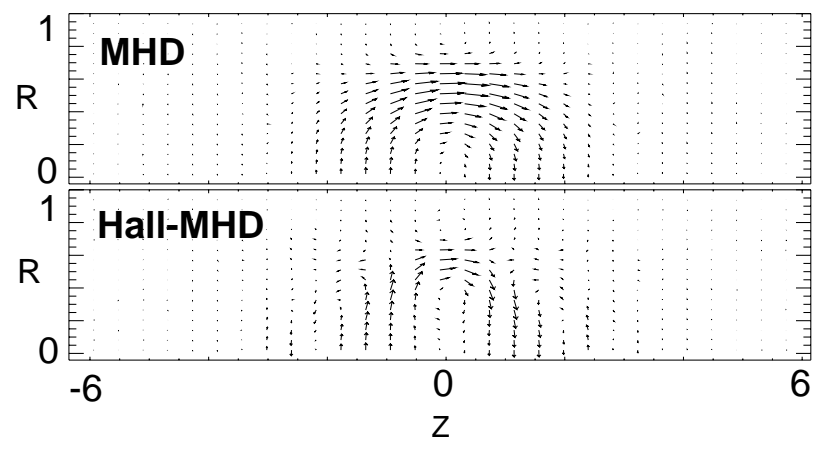

Figure 2.

Belova, Physics of Plasmas 

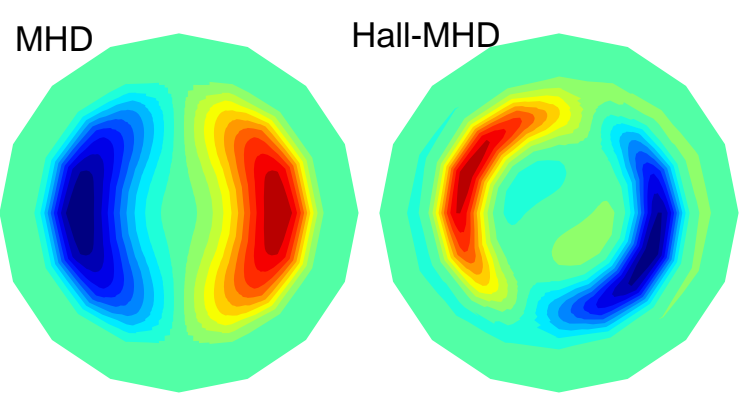

Figure 3.

Belova, Physics of Plasmas 

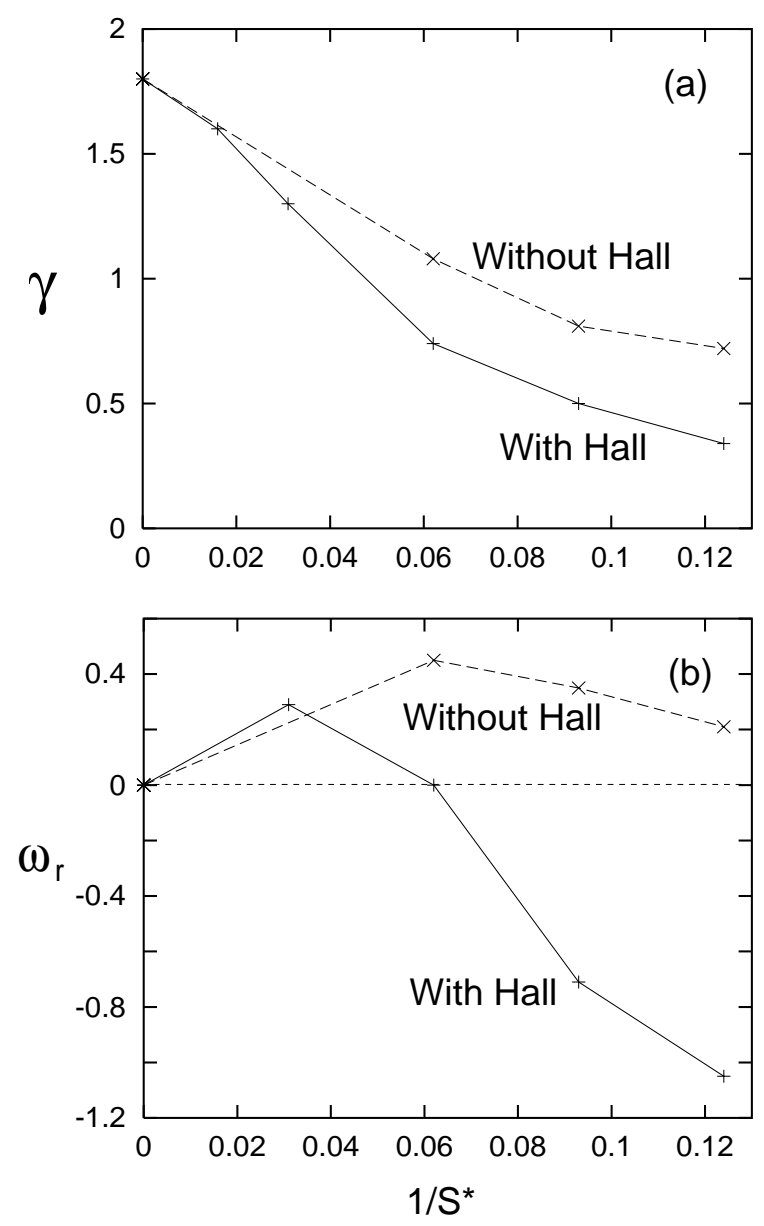

Figure 4.

Belova, Physics of Plasmas 


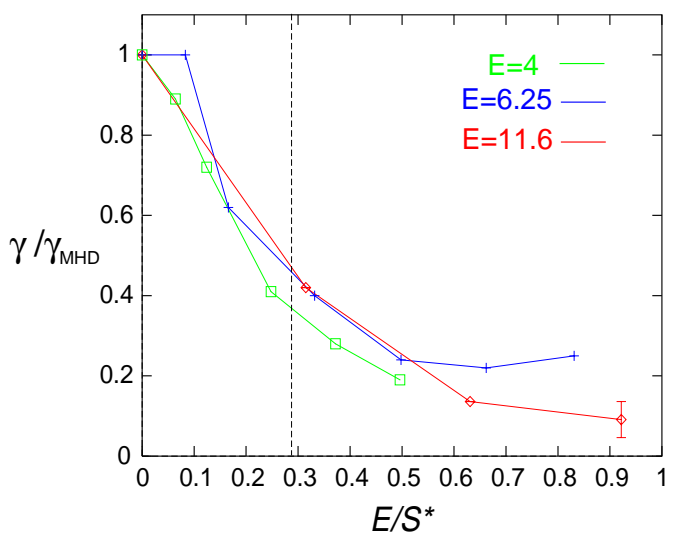

Figure 5.

Belova, Physics of Plasmas 


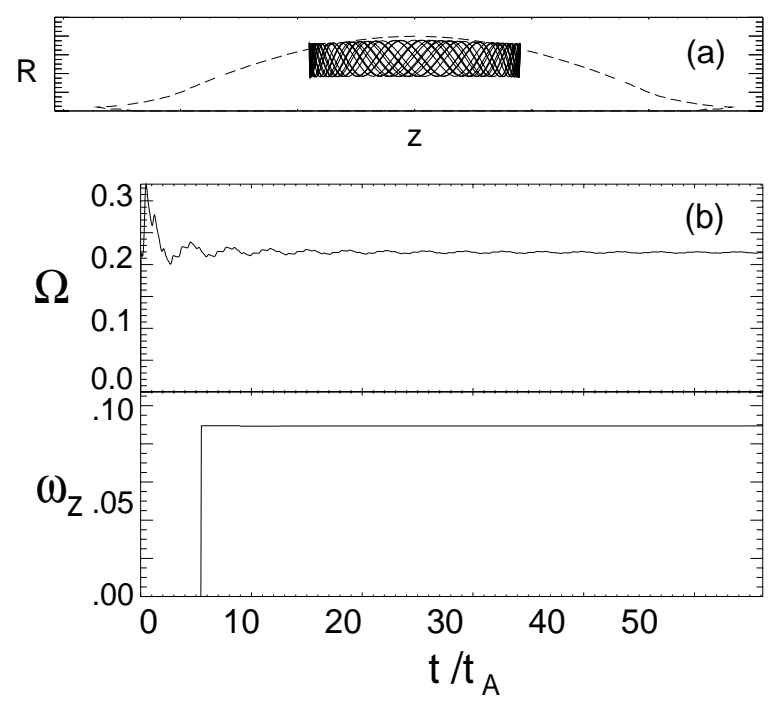

Figure 6.

Belova, Physics of Plasmas 

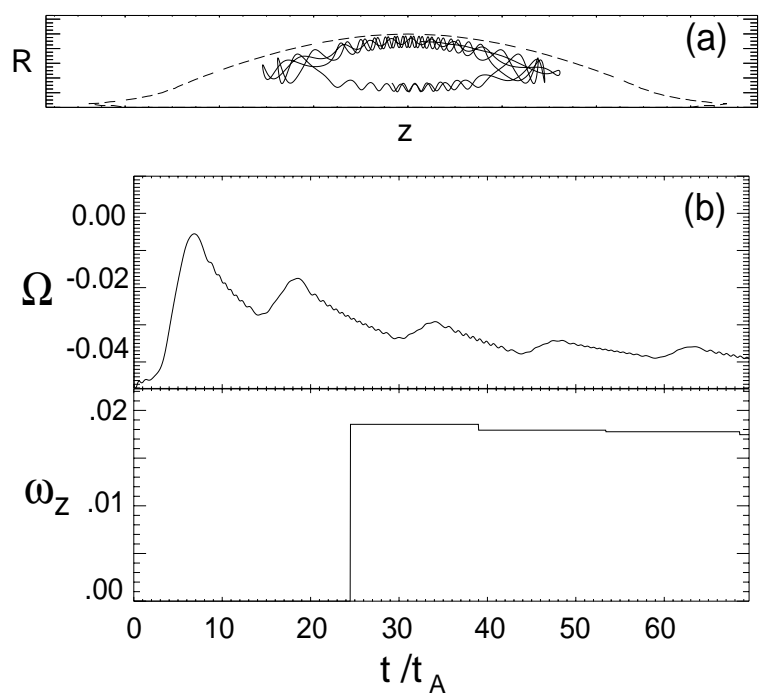

Figure 7.

Belova, Physics of Plasmas 

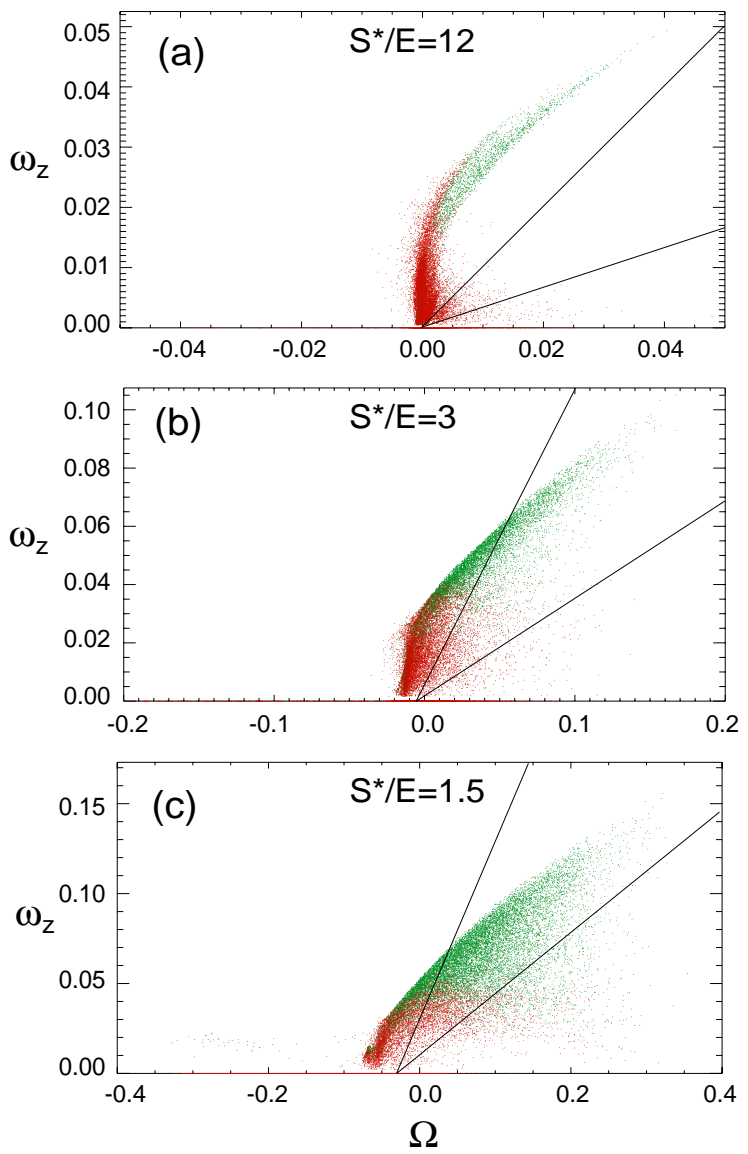

Figure 8.

Belova, Physics of Plasmas 


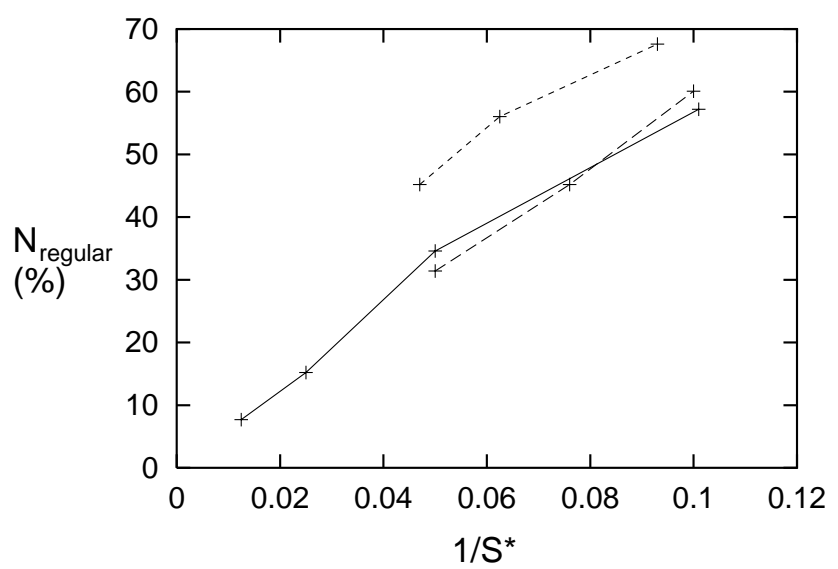

Figure 9.

Belova, Physics of Plasmas 

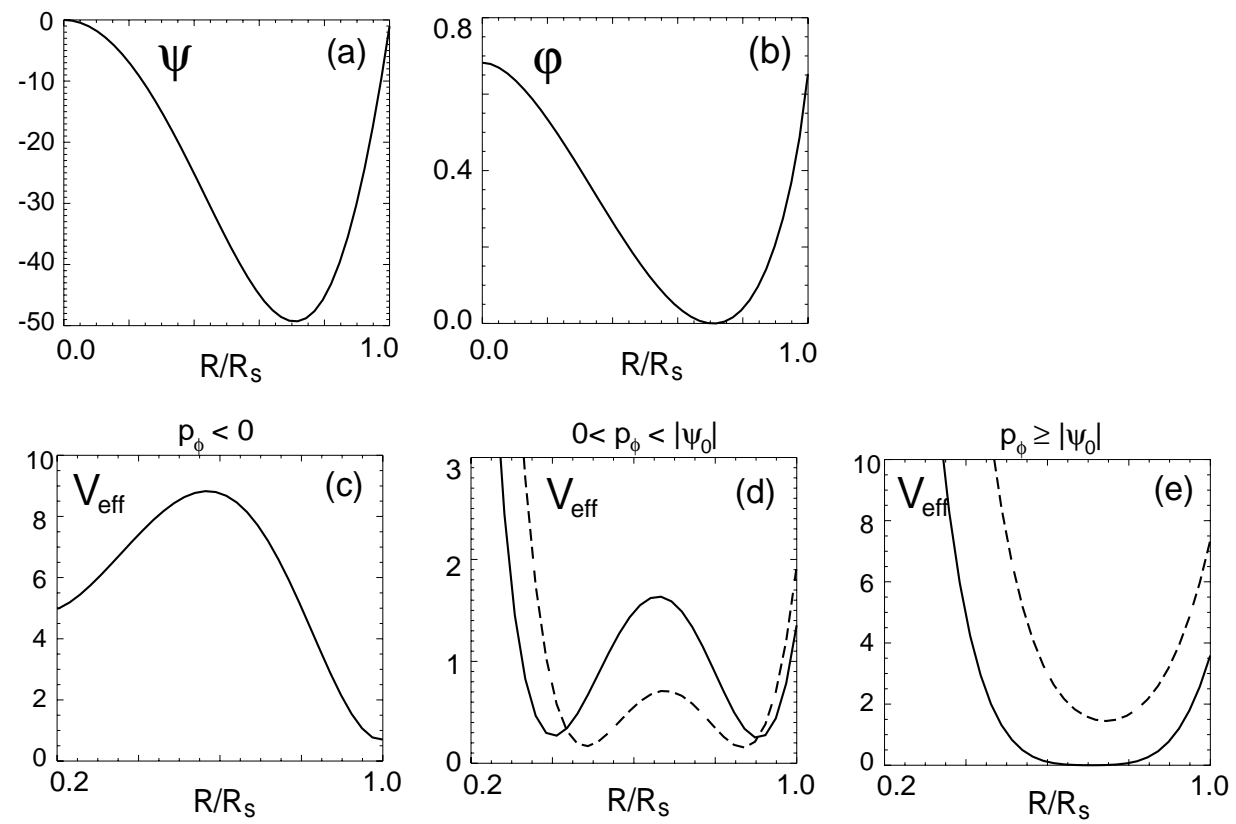

Figure 10.

Belova, Physics of Plasmas 


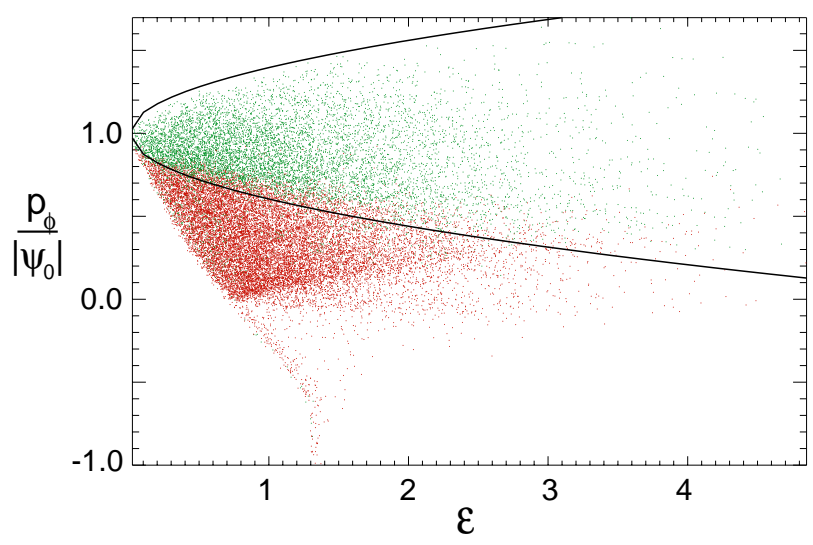

Figure 11.

Belova, Physics of Plasmas 


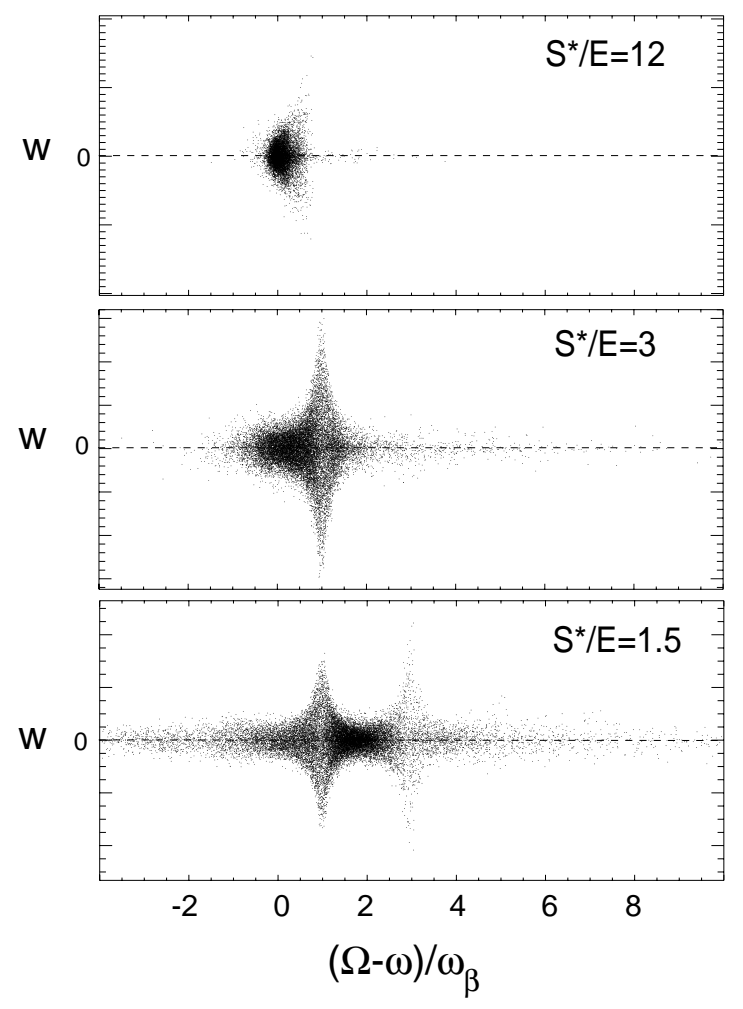

Figure 12.

Belova, Physics of Plasmas 


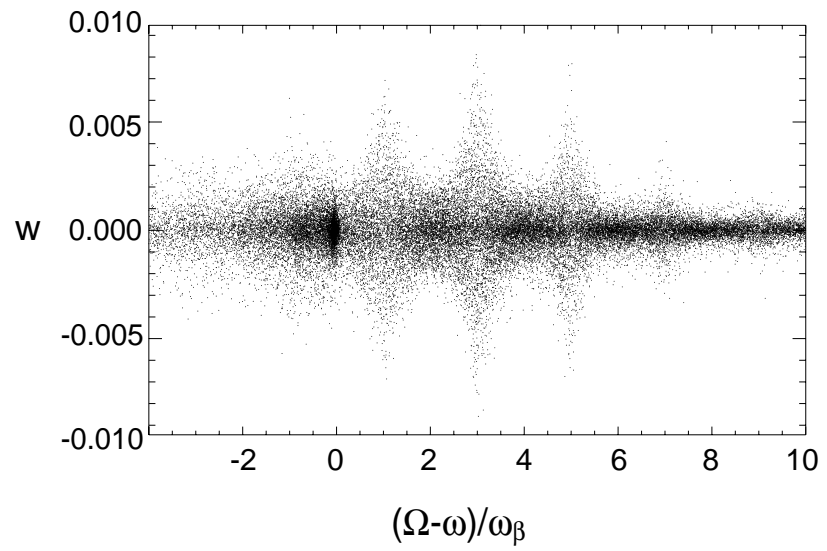

Figure 13.

Belova, Physics of Plasmas 


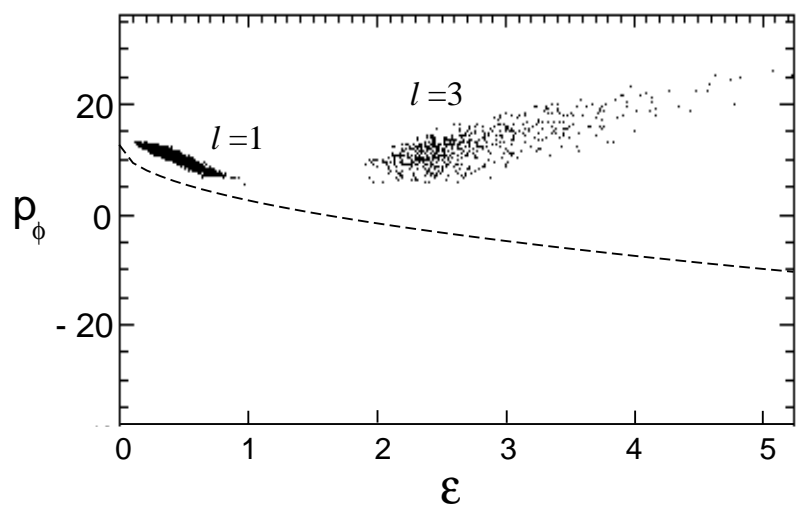

Figure 14.

Belova, Physics of Plasmas 


\section{External Distribution}

Plasma Research Laboratory, Australian National University, Australia

Professor I.R. Jones, Flinders University, Australia

Professor João Canalle, Instituto de Fisica DEQ/IF - UERJ, Brazil

Mr. Gerson O. Ludwig, Instituto Nacional de Pesquisas, Brazil

Dr. P.H. Sakanaka, Instituto Fisica, Brazil

The Librarian, Culham Laboratory, England

Mrs. S.A. Hutchinson, JET Library, England

Professor M.N. Bussac, Ecole Polytechnique, France

Librarian, Max-Planck-Institut für Plasmaphysik, Germany

Jolan Moldvai, Reports Library, MTA KFKI-ATKI, Hungary

Dr. P. Kaw, Institute for Plasma Research, India

Ms. P.J. Pathak, Librarian, Insitute for Plasma Research, India

Ms. Clelia De Palo, Associazione EURATOM-ENEA, Italy

Dr. G. Grosso, Instituto di Fisica del Plasma, Italy

Librarian, Naka Fusion Research Establishment, JAERI, Japan

Library, Plasma Physics Laboratory, Kyoto University, Japan

Research Information Center, National Institute for Fusion Science, Japan

Dr. O. Mitarai, Kyushu Tokai University, Japan

Library, Academia Sinica, Institute of Plasma Physics, People's Republic of China

Shih-Tung Tsai, Institute of Physics, Chinese Academy of Sciences, People's Republic of China

Dr. S. Mirnov, TRINITI, Troitsk, Russian Federation, Russia

Dr. V.S. Strelkov, Kurchatov Institute, Russian Federation, Russia

Professor Peter Lukac, Katedra Fyziky Plazmy MFF UK, Mlynska dolina F-2, Komenskeho Univerzita, SK-842 15 Bratislava, Slovakia

Dr. G.S. Lee, Korea Basic Science Institute, South Korea

Institute for Plasma Research, University of Maryland, USA

Librarian, Fusion Energy Division, Oak Ridge National Laboratory, USA

Librarian, Institute of Fusion Studies, University of Texas, USA

Librarian, Magnetic Fusion Program, Lawrence Livermore National Laboratory, USA

Library, General Atomics, USA

Plasma Physics Group, Fusion Energy Research Program, University of California at San Diego, USA

Plasma Physics Library, Columbia University, USA

Alkesh Punjabi, Center for Fusion Research and Training, Hampton University, USA

Dr. W.M. Stacey, Fusion Research Center, Georgia Institute of Technology, USA

Dr. John Willis, U.S. Department of Energy, Office of Fusion Energy Sciences, USA

Mr. Paul H. Wright, Indianapolis, Indiana, USA 
The Princeton Plasma Physics Laboratory is operated by Princeton University under contract with the U.S. Department of Energy.

\author{
Information Services \\ Princeton Plasma Physics Laboratory \\ P.O. Box 451 \\ Princeton, NJ 08543
}

Phone: 609-243-2750

Fax: 609-243-2751

e-mail: pppl_info@pppl.gov

Internet Address: http://www.pppl.gov 